\title{
Numerical Modeling of Two-Phase Gravitational Granular Flows with Bottom Topography
}

\author{
Marica Pelanti ${ }^{1}$, François Bouchut ${ }^{1}$, Anne-Mangeney-Castelnau ${ }^{2}$ and \\ Jean-Pierre Vilotte ${ }^{2}$ \\ 1 Département de Mathématiques et Applications, École Normale Supérieure, \\ 45, rue d'Ulm - 75230 Paris cedex 05, France. Marica.Pelanti@ens.fr \\ (M. Pelanti), Francois.Bouchut@ens.fr (F. Bouchut). \\ 2 Département de Sismologie, Institut de Physique du Globe de Paris, 4, place \\ Jussieu - 75252 Paris cedex 05, France. mangeney@ipgp.jussieu.fr \\ (A. Mangeney-Castelnau), vilotte@ipgp.jussieu.fr (J.-P. Vilotte).
}

Summary. We study a depth-averaged model of gravity-driven mixtures of solid grains and fluid moving over variable basal surface. The particular application we are interested in is the numerical description of geophysical flows such as avalanches and debris flows, which typically contain both solid material and interstitial fluid. The depth-averaged mass and momentum equations for the solid and fluid components form a non-conservative system, where non-conservative terms involving the derivatives of the unknowns couple together the sets of equations of the two phases. The system can be shown to be hyperbolic at least when the difference of velocities of the two constituents is sufficiently small.

We numerically solve the model equations in one dimension by a finite volume scheme based on a Roe-type Riemann solver. Well-balancing of topography source terms is obtained via a technique that includes these contributions into the wave structure of the Riemann solution.

\section{Introduction}

Geophysical flows such as avalanches and debris flows are gravity-driven granular masses, typically composed of a mixture of solid grains and interstitial fluid. Following the pioneering work of Savage and Hutter [SH89], in recent years great advances have been made in the mathematical and numerical modeling of these natural processes by means of depth-averaged (thin layer) models, which are based on the small aspect ratio of typical flows. Early studies [SH89, SH91], and part of recent literature as well [MCVB ${ }^{+}$03, PH03], were limited to dry (one-phase) granular flows. Iverson [Ive97] and Iverson and Denlinger [ID01] first addressed the need of accounting for interstitial 
fluid effects in the flowing mass, and developed a solid-fluid mixture theory based on the simplifying assumptions of constant porosity and equality of fluid and solid velocities. See also later extensions [DI04] and similar approaches [PWH05]. Making a step forward, Pitman and Le [PL05] have recently presented a depth-averaged two-phase model for debris flows and avalanches that contains mass and momentum equations for both the solid and fluid component, thus providing equations for porosity and velocity of both phases. However, in this work the authors propose a numerical method only for a reduced model that ignores fluid inertial terms.

With the objective of contributing to develop a numerical model describing accurately the co-existence and the interaction between solid and fluid constituents, here we study a two-phase granular flow model that follows the work of [PL05]. The mathematical equations are presented in Section 2, and in Section 3 we analyze eigenvalues and hyperbolicity. Then, in Section 4, we illustrate the numerical technique we use to solve the model system, and we report some numerical results in Section 5 . Some prospected work is finally mentioned in Section 6 .

\section{Mathematical model}

Following Pitman and Le [PL05], we consider a thin layer of a mixture of solid granular material and fluid moving over a smooth basal surface. Solid and fluid components are assumed incompressible, with constant specific densities $\rho_{s}$ and $\rho_{f}<\rho_{s}$, respectively. Under the shallow flow assumption, depthaveraged mass and momentum equations for the two phases can be derived in the following form (here in one dimension):

$$
\begin{aligned}
& \partial_{t}(\varphi h)+\partial_{x}\left(\varphi h v_{s}\right)=0 \\
& \partial_{t}\left(\varphi h v_{s}\right)+\partial_{x}\left(\varphi h v_{s}^{2}+\frac{g}{2}(1-r) \varphi h^{2}\right) \\
& \quad=-r \frac{g}{2} \varphi \partial_{x} h^{2}-g \varphi h \partial_{x} b+\nu_{x z} g(1-r) \varphi h+r D h\left(v_{f}-v_{s}\right) \\
& \partial_{t}((1-\varphi) h)+\partial_{x}\left((1-\varphi) h v_{f}\right)=0 \\
& \partial_{t}\left((1-\varphi) h v_{f}\right)+\partial_{x}\left((1-\varphi) h v_{f}^{2}\right) \\
& \quad=-(1-\varphi) \frac{g}{2} \partial_{x} h^{2}-g(1-\varphi) h \partial_{x} b-D h\left(v_{f}-v_{s}\right)
\end{aligned}
$$

Here $h$ is the flow depth, $\varphi$ the solid volume fraction, $v_{s}$ and $v_{f}$ are the solid and fluid velocities, respectively, and $b(x)$ represents the bottom topography. Moreover, $D$ is a drag function, $\nu_{x z}$ a friction coefficient, $g$ the gravity constant, and $r=\frac{\rho_{f}}{\rho_{s}}<1$. The two-phase model (1) is a variant of the Pitman-Le model [PL05], and it differs from the original work of [PL05] in the description of the fluid and mixture momentum balance. See [BPMC] for more details. In particular, contrarily to [PL05], our model has the property of recovering a conservative equation for the momentum of the mixture, which has the form: 


$$
\begin{aligned}
& \partial_{t}\left(\varphi h v_{s}+r(1-\varphi) h v_{f}\right)+\partial_{x}\left(\varphi h v_{s}^{2}+r(1-\varphi) h v_{f}^{2}+\frac{g}{2}(\varphi+r(1-\varphi)) h^{2}\right) \\
& =-g(\varphi+r(1-\varphi)) h \partial_{x} b+\nu_{x z} g(1-r) \varphi h
\end{aligned}
$$

\subsection{Formulation in $h_{s}, h_{f}$}

Let us rewrite our model in terms of the variables $h_{s}=\varphi h$ and $h_{f}=(1-\varphi) h$. We neglect friction and drag, which will not be considered hereafter. Modeling of drag will be discussed in [BPMC]. Setting $q=\left(h_{s}, h_{s} v_{s}, h_{f}, h_{f} v_{f}\right)^{\mathrm{T}}$, we have the system

$$
\begin{gathered}
\partial_{t} q+\partial_{x} f(q)+s\left(q, \partial_{x} q\right)=\psi^{\mathrm{b}}(q), \quad \text { with } \\
f(q)=\left(h_{s} v_{s}, h_{s} v_{s}^{2}+\frac{g}{2} h_{s}^{2}+\frac{g(1-r)}{2} h_{s} h_{f}, h_{f} v_{f}, h_{f} v_{f}^{2}+\frac{g}{2} h_{f}^{2}\right)^{\mathrm{T}}, \\
s\left(q, \partial_{x} q\right)=\left(0, r g h_{s} \partial_{x} h_{f}, 0, g h_{f} \partial_{x} h_{s}\right)^{\mathrm{T}} \\
\text { and } \psi^{\mathrm{b}}(q)=-\left(0, g h_{s} \partial_{x} b, 0, g h_{f} \partial_{x} b\right)^{\mathrm{T}} .
\end{gathered}
$$

Above, we have put into evidence the conservative portion of the system $\frac{\partial f(q)}{\partial x}$, and the non-conservative term $s\left(q, \partial_{x} q\right)$. An interesting feature of this formulation of our model in $h_{s}, h_{f}$ is that it shows similarity with the classical two-layer shallow water model. The only difference is the additional conservative cross term $\frac{\partial}{\partial x}\left(\frac{g}{2}(1-r) h_{s} h_{f}\right)$ in the solid momentum equation of our two-phase system. Let us finally write system (3) in quasi-linear form. We have $\partial_{t} q+A(q) \partial_{x} q=\psi^{\mathrm{b}}(q)$, where

$$
A(q)=\left(\begin{array}{cccc}
0 & 1 & 0 & 0 \\
-v_{s}^{2}+g h_{s}+\frac{g(1-r)}{2} h_{f} & 2 v_{s} & \frac{g(1+r)}{2} h_{s} & 0 \\
0 & 0 & 0 & 1 \\
g h_{f} & 0 & -v_{f}^{2}+g h_{f} & 2 v_{f}
\end{array}\right) .
$$

\section{Eigenvalue analysis and hyperbolicity}

In general, simple explicit expressions of the eigenvalues $\lambda_{k}, k=1, \ldots, 4$, of the matrix $A$ of the system can not be found. In the particular case of equality of solid and fluid velocities, $v_{f}=v_{s} \equiv v$, the eigenvalues are real and distinct $(\varphi \neq 1)$, and given by $\lambda_{1,4}=v \mp a$, and $\lambda_{2,3}=v \mp a \beta$, where we have introduced the quantities

$$
a=\sqrt{g h} \quad \text { and } \quad \beta=\sqrt{\frac{1}{2}(1-\varphi)(1-r)}<1 .
$$

Other particular cases are: (i) $\varphi=0$, for which the eigenvalues are $v_{f} \mp a$, $v_{s} \mp a \beta$, with $\beta=\sqrt{\frac{1-r}{2}}$; (ii) $\varphi=1$, for which we find the two distinct eigenvalues $v_{s} \mp a$ and the double eigenvalue $v_{f}$.

In general, for $h>0$, we can state the following result: 
Proposition 1. Matrix $A$ has always at least two real eigenvalues $\lambda_{1,4}$, and moreover, the eigenvalues $\lambda_{k}$ of $A, k=1, \ldots, 4$, satisfy:

$$
\min \left(v_{f}, v_{s}\right)-a \leq \lambda_{1} \leq \mathfrak{R e}\left(\lambda_{2}\right) \leq \mathfrak{R e}\left(\lambda_{3}\right) \leq \lambda_{4} \leq \max \left(v_{f}, v_{s}\right)+a,
$$

where $\mathfrak{R e}(\cdot)$ denotes the real part. Furthermore:

(i) If $\left|v_{s}-v_{f}\right| \leq 2 a \beta$ or $\left|v_{s}-v_{f}\right| \geq 2 a$ then all the eigenvalues are real. If these inequalities are strictly satisfied, and if $\varphi \neq 1$, then the eigenvalues are also distinct, and system (3) is strictly hyperbolic.

(ii) If $2 a \beta<\left|v_{s}-v_{f}\right|<2 a$ then the internal eigenvalues $\lambda_{2,3}$ may be complex.

For the proof we refer to [BPMC].

\subsection{Eigenvectors}

The right and left eigenvectors of the matrix $A(q)$ can be easily written in terms of the eigenvalues $\lambda_{k}$. Let us assume here $h_{s}, h_{f}>0$. Then the right eigenvectors $r_{k}, k=1, \ldots, 4$, can be expressed as $r_{k}=\left(1, \lambda_{k}, b_{k}, b_{k} \lambda_{k}\right)^{\mathrm{T}}$, with $b_{k}=2 \frac{\left(\lambda_{k}-v_{s}\right)^{2}-g\left(h_{s}+h_{f}(1-r) / 2\right)}{g(1+r) h_{s}}=\frac{g h_{f}}{\left(\lambda_{k}-v_{f}\right)^{2}-g h_{f}}$. The left eigenvectors $l_{k}$ of $A$ can be taken as $l_{k}=\frac{n_{k}}{P^{\prime}\left(\lambda_{k}\right)}$, where $P(\lambda)$ is the characteristic polynomial of $A$ and $n_{k}=\left(c_{s, k}\left(\lambda_{k}-2 v_{s}\right), c_{s, k}, c_{f}\left(\lambda_{k}-2 v_{f}\right), c_{f}\right)$, with $c_{s, k}=\left(\lambda_{k}-v_{f}\right)^{2}-g h_{f}$ and $c_{f}=g \frac{(1+r)}{2} h_{s}$. Here we have normalized the eigenvectors $l_{k}$ so that $L=R^{-1}$, where $R$ is the matrix with columns $r_{k}$, and $L$ the matrix with rows $l_{k}$.

\section{Numerical solution}

We assume $h_{s}, h_{f}>0$ during the flow evolution, and that the difference between solid and fluid velocities is small enough so that the model system is strictly hyperbolic. We develop a numerical solution method for (3) in the framework of finite volume schemes based on Riemann solvers.

\subsection{A Roe-type scheme}

Let us first consider system (3) without topography terms, $\partial_{t} q+\partial_{t} f(q)+$ $s\left(q, \partial_{x} q\right)=0$. We numerically solve these equations by employing a Roe-type [Roe81] method. Following the usual technique, at every time step and at each interface between left and right states $q_{\ell}, q_{r}$, we solve a Riemann problem for a linearized system $\partial_{t} q+\hat{A}\left(q_{\ell}, q_{r}\right) \partial_{x} q=0$. The constant coefficient matrix $\hat{A}\left(q_{\ell}, q_{r}\right)$ is defined so as to guarantee conservation for the mass of each phase and for the momentum of the mixture. That is, we need $\Delta f^{(p)}=\hat{A}_{(p,:)} \Delta q$, for $p=1$ and $p=3$, and $\Delta f^{(2)}+r \Delta f^{(4)}=\left(\hat{A}_{(2,:)}+r \hat{A}_{(4,:)}\right) \Delta q$, where $\Delta q=q_{r}-q_{\ell}$, and $\Delta f=f\left(q_{r}\right)-f\left(q_{\ell}\right)$. This can be satisfied by taking $\hat{A}$ as 
the original matrix $A(q)$ evaluated in an average state $\hat{q}=\hat{q}\left(\hat{h}_{s}, \hat{h}_{f}, \hat{v}_{s}, \hat{v}_{f}\right)$, where

$$
\hat{h}_{\iota}=\frac{h_{\iota, \ell}+h_{\iota, r}}{2} \quad \text { and } \quad \hat{v}_{\iota}=\frac{\sqrt{h_{\iota, \ell}} v_{\iota, \ell}+\sqrt{h_{\iota, r}} v_{\iota, r}}{\sqrt{h_{\iota, \ell}}+\sqrt{h_{\iota, r}}}, \quad \iota=s, f .
$$

\subsection{F-wave formulation}

The algorithm we employ is formulated in the framework of the wavepropagation methods of [LeV97]. In particular, we adopt the so-called f-wave formulation [BLMR02] of these methods, since this approach will be useful to incorporate topography source terms (see next section).

The f-wave technique is designed for conservative systems, that is systems endowed with a flux function, and its main idea is to define the structure of the Riemann solution by decomposing into waves (f-waves) the flux difference between neighboring cells. Although our system (3) contains the non-conservative products $s\left(q, \partial_{x} q\right)$, we can still employ this approach by defining locally an approximate flux $\tilde{f}$ consistent with the Roe linearization. Here we take $\tilde{f}(q)=f(q)+\left(0, r g \hat{h}_{s} h_{f}, 0, g \hat{h}_{f} h_{s}\right)^{\mathrm{T}}$, with $\hat{h}_{s}, \hat{h}_{f}$ as in (7). Note that $\Delta \tilde{f}=\hat{A} \Delta q$. Then, in our algorithm we project the difference $\Delta \tilde{f}=\tilde{f}\left(q_{r}\right)-\tilde{f}\left(q_{\ell}\right)$ onto the eigenvectors $\hat{r}_{k}$ of the Roe matrix, $\Delta \tilde{f}=\sum_{k=1}^{4} \zeta_{k} \hat{r}_{k}$, and we use the f-waves $\mathcal{Z}_{k} \equiv \zeta_{k} \hat{r}_{k}$ with corresponding speeds $\hat{\lambda}_{k}$ (eigenvalues of $\hat{A}$ ) to update cell averages. Second-order correction terms and limiters are applied to these f-waves. See [LeV97, BLMR02, LeV02] for details.

\subsection{Topography source terms}

We now consider system (3) with bottom topography source terms included. A well known difficulty in the approximation of hyperbolic systems with sources (e.g. [Bou04]) is the preservation of steady state conditions at the discrete level, and the efficient modeling of small perturbations from steady states. In particular, for the system under study we are concerned with the steady state conditions at rest $h+b=$ const., $\varphi=$ const., $v_{s}=v_{f}=0$. To build a well-balanced scheme, we follow the approach of [BLMR02, LP01, LG04], which uses the f-wave formulation framework described above. The idea is to incorporate the effect of bottom topography terms into the Riemannn solution, by taking interface values $\Psi_{\ell, r}^{\mathrm{b}}$ of the topography source term $\psi^{\mathrm{b}}(q)$ and by including contributions $\Psi_{\ell, r}^{\mathrm{b}} \Delta x$ into the wave splitting that we have defined for the solution of the homogeneous system. We now decompose $\Delta \tilde{f}-\Psi_{\ell, r}^{\mathrm{b}} \Delta x=\sum_{k=1}^{4} \zeta_{k} \hat{r}_{k}$. The interface source term $\Psi_{\ell, r}^{\mathrm{b}}$ must be defined so that the discrete steady state condition $\Delta \tilde{f} / \Delta x=\Psi_{\ell, r}^{\mathrm{b}}$ holds whenever initial Riemann data correspond to equilibrium, that is $(h+b)_{\ell}=(h+b)_{r}$, $\varphi_{\ell}=\varphi_{r}, v_{s, \ell}=v_{s, r}=v_{f, \ell}=v_{f, r}=0$. To satisfy this requirement we take $\Psi_{\ell, r}^{\mathrm{b}} \Delta x=\left(0, g \hat{h}_{s} \Delta b, 0, g \hat{h}_{f} \Delta b\right)^{\mathrm{T}}$, with $\Delta b=b_{r}-b_{\ell}$. 


\section{A numerical test: perturbation of a steady state}

We have implemented our algorithm by using the basic Fortran 77 routines of the CLAWPACK software [LeV]. Let us mention that, since explicit formulas for the $\hat{\lambda}_{k}$ s are not available, the external eigenvalues $\hat{\lambda}_{1,4}$ are computed numerically through Newton's iteration method. The internal eigenvalues $\hat{\lambda}_{2,3}$ can be then found analytically.

We present here the results of a numerical experiment that is an extension of LeVeque's classical test [LeV98] for (one-phase) shallow water equations with bottom topography. In this problem we observe the behavior of a small perturbation of steady state conditions at rest over a bottom topography defined by $b(x)=0.25(\cos (\pi(x-0.5) / 0.1)+1)$ if $x \in(0.4,0.6)$, and $b(x)=0$ otherwise. Initially, we take a small perturbation of the flow depth $h$ and of the solid volume fraction $\varphi: h(x, 0)=h_{0}+\tilde{h}$ and $\varphi(x, 0)=\varphi_{0}-\tilde{\varphi}$ for $x \in$ $(-0.6,-0.5)$, with $h_{0}=1, \varphi_{0}=0.6$, and $\tilde{h}=\tilde{\varphi}=10^{-3}$. The computational domain is $[-0.9,1.1]$, and free flow boundary conditions are used. Moreover, we take $r=1 / 2$ and $g=1$. We compute the solution with 100 grid cells and compare it with a fine grid reference solution obtained with 1000 grid cells. Second order corrections are used to achieve high resolution.

In Figure 1 we display results at four different times for $h+b$ and $\varphi$ (top and bottom subplot of each subfigure, respectively). The bold line over the $\mathrm{x}$ interval $[0.4,0.6]$ in the plots of $h+b$ indicates the region of the domain where $b(x) \neq 0$. As we can observe from the first couple of plots (subfig. (a)), the initial perturbation splits into four waves. These are approximately linear waves propagating at the characteristic speeds corresponding to the background state, that is $\pm \sqrt{g h_{0}}$ for the external waves, and $\pm \sqrt{\frac{g}{2} h_{0}\left(1-\varphi_{0}\right)(1-r)}$ for the internal ones. Let us remark that in this problem $\varphi$ appears to vary only across the internal waves as a consequence of the initial conditions, for which in particular $v_{s}=v_{f}$. The second couple of plots of $h+b$ and $\varphi$ (subfig. (b)) shows the time at which the right-going external wave has just passed over the obstacle at the bottom, and it has been partially reflected. Similarly, the third couple of plots (subfig. (c)) shows when the right-going internal wave has now moved past the hump and has produced a reflected wave. At this time the reflected wave generated by the external wave has left the domain from the left boundary, after passing through the incoming internal wave. The last plots (subfig. (d)) show the situation in which all the waves have exited from the domain, except the disturbance produced by the internal wave, which will eventually leave from the left boundary. No spurious disturbances are observed in this test.

\section{Prospected work}

We have presented a numerical model of grain-fluid mixtures over variable topography. This is only a first stage towards the development of a model 

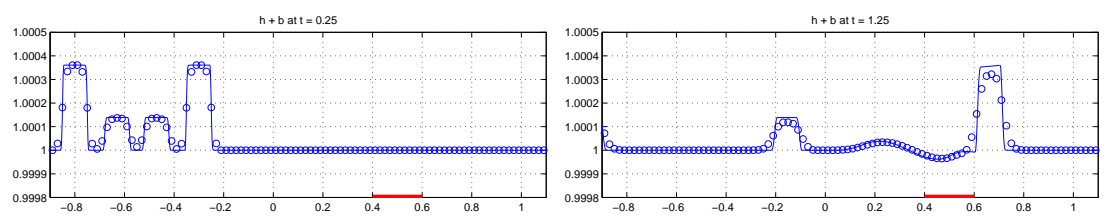

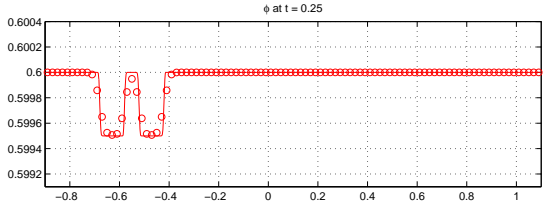

(a) $t=0.25$

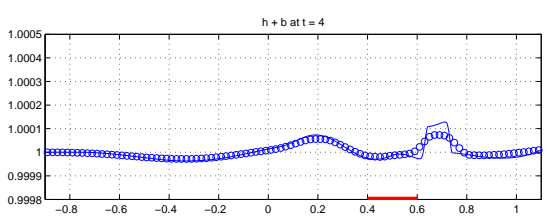

$\phi$ at $t=4$

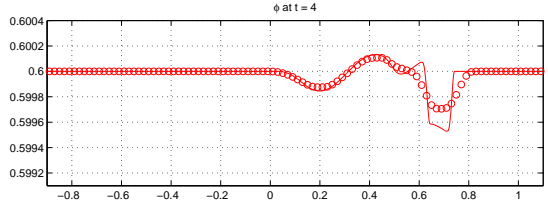

(c) $t=4.0$

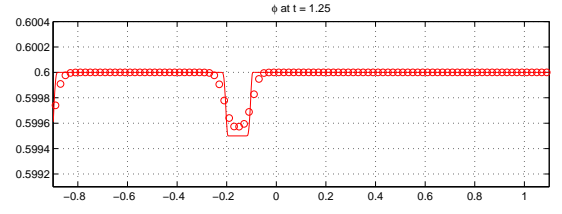

(b) $t=1.25$

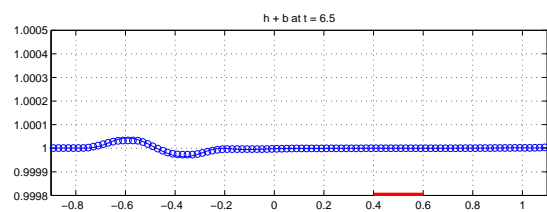

att $=6.5$

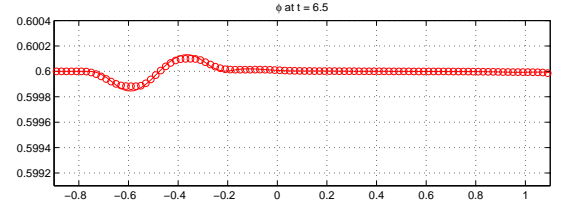

(d) $t=6.5$

Fig. 1. Numerical test of Section 5. Solution for $h+b$ and $\varphi$ (top and bottom subplot of each subfigure). Circles: solution computed with 100 grid cells; continuous line: reference solution with 1000 grid cells.

applicable to realistic geophysical flows. The primary issue on which we are currently focusing our research efforts is preservation of positivity of the flow depth $(h \geq 0)$, to be able to handle interfaces between flow fronts and bed dry states. Planned work includes also the extension of the model to arbitrary complex topography, and implementation of a two-dimensional scheme.

\section{Acknowledgments}

Part of this work was done by M. Pelanti during a post-doctoral stay at the Institut du Globe de Paris financed by the City of Paris.

\section{References}

[BLMR02] D. Bale, R. J. LeVeque, S. Mitran, and J. A. Rossmanith. A wavepropagation method for conservation laws and balance laws with spa- 

F. Bouchut. Nonlinear stability of finite volume methods for hyperbolic conservation laws, and well-balanced schemes for sources. BirkhäuserVerlag, 2004.

[BPMC $]$ F. Bouchut, M. Pelanti, and A. Mangeney-Castelnau. A Roe-type scheme for two-phase granular flows with bottom topography. In Preparation.

[DI04] R. P. Denlinger and R. M. Iverson. Granular avalanches across irregular three-dimensional terrain: 1. Theory and computation. J. Geophys. Res., 109:F01014, 2004.

[ID01] R. M. Iverson and R. P. Denlinger. Flow of variably fluidized granular masses across three-dimensional terrain: 1 . Coulomb mixture theory. $J$. Geophys. Res., 106:537-552, 2001.

[Ive97] R. M. Iverson. The physics of debris flows. Rev. Geophys., 35:245-296, 1997.

[LeV] R. J. LeVeque. ClAWPACK software. http: //www . amath. washington. edu/ claw.

[LeV97] R. J. LeVeque. Wave propagation algorithms for multi-dimensional hyperbolic systems. J. Comput. Phys., 131:327-353, 1997.

[LeV98] R. J. LeVeque. Balancing source terms and flux gradients in highresolution Godunov methods: The quasi-steady wave-propagation algorithm. J. Comput. Phys., 146:346-365, 1998.

[LeV02] R. J. LeVeque. Finite Volume Methods for Hyperbolic Problems. Cambridge University Press, 2002.

[LG04] R. J. LeVeque and D. L. George. High-resolution finite volume methods for the shallow water equations with bathymetry and dry states. In P. Liu, editor, Proceedings of Long-Wave Workshop, Catalina, page to appear, 2004.

[LP01] R. J. LeVeque and M. Pelanti. A class of approximate Riemann solvers and their relation to relaxation schemes. J. Comput. Phys., 172:572$591,2001$.

$\left[\mathrm{MCVB}^{+}\right.$03] A. Mangeney-Castelnau, J.-P. Vilotte, M. O. Bristeau, B. Perthame, F. Bouchut, C. Simeoni, and S. Yernini. Numerical modeling of avalanches based on Saint-Venant equations using a kinetic scheme. J. Geophys. Res., 108(B11):2527, 2003.

[PH03] S. P. Pudasaini and K. Hutter. Rapid shear flows of dry granular masses down curved and twisted channels. J. Fluid Mech., 495:193-208, 2003.

[PL05] E. B. Pitman and L. Le. A two-fluid model for avalanche and debris flows. Phil. Trans. R. Soc. A, 363:1573-1601, 2005.

[PWH05] S. P. Pudasaini, Y. Wang, and K. Hutter. Modelling debris flows down general channels. Natural Hazards and Earth System Sciences, 5:799819, 2005.

[Roe81] P. L. Roe. Approximate Riemann solvers, parameter vectors, and difference schemes. J. Comput. Phys., 43:357-372, 1981.

[SH89] W.Z. Savage and K. Hutter. The motion of a finite mass of granular material down a rough incline. J. Fluid. Mech., 199:177-215, 1989.

[SH91] S. B. Savage and K. Hutter. The dynamics of avalanches of granular materials from initiation to runout, part I. Analysis. Acta Mech., 86:201-223, 1991. 